\title{
Doenças negligenciadas e indústria farmacêutica: o caso da Doença de Chagas
}

\author{
Neglected diseases and pharmaceutical industry: the case of Chagas Disease \\ Enfermedades desatendidas y la industria farmacéutica: el caso de la Enfermedad de Chagas
}

Recebido: 27/01/2021 | Revisado: 02/02/2021 | Aceito: 05/02/2021 | Publicado: 10/02/2021

\begin{abstract}
Alison Pontes da Silva
ORCID: https://orcid.org/0000-0002-9662-4111

Universidade Federal de Campina Grande, Brasil E-mail: alison.pontes@estudante.ufcg.edu.br

Gabriel Magno Santos Dantas ORCID: https://orcid.org/0000-0001-9505-5714 Universidade Federal de Campina Grande, Brasil E-mail: gabriel.magno@estudante.ufcg.edu.br

Pedro Ithalo Francisco da Silva ORCID: https://orcid.org/0000-0002-8976-2591 Universidade Federal de Campina Grande, Brasil E-mail: pedro.ithalo@estudante.ufcg.edu.br

Andressa Nayara Gomes de Medeiros ORCID: https://orcid.org/0000-0002-4780-3279 Universidade Federal de Campina Grande, Brasil E-mail: andressa.nayara@estudante.ufcg.edu.br Toshiyuki Nagashima Junior ORCID: https://orcid.org/0000-0002-5131-1058 Universidade Federal de Campina Grande, Brasil E-mail: nagashima@ufcg.edu.br
\end{abstract}

\begin{abstract}
Resumo
O desenvolvimento de novos fármacos, no contexto das Doenças Tropicais Negligenciadas (DTNs), permanece como um grande desafio. Dentre as DTNs, destaca-se a Doença de Chagas (DC), a qual possui elevada morbimortalidade. Assim, este estudo objetiva resgatar informações acerca do cenário atual, bem como novas estratégias e inovações no tratamento farmacológico da DC. Para tal, foi realizada uma revisão narrativa, em que foram selecionados artigos no PubMed, SciELO e LILACS, no período entre 2015 e 2020. Buscando avaliar a relevância no meio acadêmico, comparou-se o número de publicações no SciELO entre 1950 e 2019, sobre DC, diabetes mellitus e câncer. Também se buscou informações sobre ensaios clínicos no site ClinicalTrials.gov. Nos últimos 20 anos, o número de publicações sobre a DC no SciELO foi bastante inferior se comparado com diabetes mellitus e câncer. Ademais, apenas dois medicamentos estão disponíveis, sendo eles benznidazol e nifurtimox, os quais possuem limitações como a quantidade de efeitos adversos. Por isso, novas opções terapêuticas (grânulos, sistemas multiparticulados, etc) têm sido estudadas, principalmente no caso do benznidazol, tendo demonstrado resultados promissores quanto à otimização de toxicidade, absorção e biodisponibilidade, por exemplo. Quanto aos ensaios clínicos, foi encontrado um total de sete estudos, os quais investigam cinco compostos para o tratamento da DC. Somente um dos compostos não tem uso clínico já registrado, o selênio, o que sugere uma deficiência na busca por novos candidatos à fármacos. Tais informações reforçam, portanto, o caráter negligenciado da DC, bem como enfatizam a importância de pesquisar novas terapias para esta parasitose.
\end{abstract}

Palavras-chave: Doenças negligenciadas; Doença de chagas; Desenvolvimento de medicamentos.

\begin{abstract}
The development of new active ingredients in the context of Neglected Tropical Diseases (NTDs), remains a big challenge to the society. Among NTDs it is worth highlight the Chagas disease (CD) which has high morbidity and mortality. This study aims to retrieve information about the current scenario as well as new strategies and innovations in the pharmacological treatment of $\mathrm{CD}$. For this purpose, a narrative review was conducted in which the articles were selected in PubMed, SciELO and LILACS, in the period between 2015/2020. Seeking to assess in order of relevance in academia the data were observed in results of publications in the SciELO between 1950/2019, about CD, diabetes mellitus and cancer. Information on clinical trials was also searched at ClinicalTrials.gov. In the last 20 years the number of publications on CD in SciELO was significantly lower compared to diabetes mellitus and cancer. Moreover, only two drugs are available, benznidazole and nifurtimox, which have limitations such as the amount of adverse effects. Therefore, new therapeutic options have been studied mainly in the case of benznidazole and have
\end{abstract}


shown promising results by optimizing toxicity, absorption and bioavailability, for example. With regard to clinical trials, a total of seven studies were found, which investigate five compounds for the treatment of CD. Only one of the compounds has no clinical use yet, selenium, which suggests a deficiency in the search for new drug candidates. Such information reinforces, therefore, the neglected character of $\mathrm{CD}$, as well as emphasizing the importance of researching new therapies.

Keywords: Neglected diseases; Chagas disease; Drug development.

\section{Resumen}

El desarrollo de nuevos medicamentos, en el contexto de las Enfermedades Tropicales Desatendidas (ETD), sigue siendo un gran desafío. Entre las ETD se destaca la Enfermedad de Chagas (EC), que presenta una alta morbilidad y mortalidad. Este estudio tiene como objetivo rescatar información sobre el escenario actual, así como nuevas estrategias e innovaciones en el tratamiento farmacológico de la EC. Para ello se realizó una revisión narrativa, que se seleccionaron artículos en PubMed, SciELO y LILACS, en el período entre 2015/2020. La relevancia en el ámbito académico se comparó el número de publicaciones en SciELO entre 1950 y 2019, sobre EC, diabetes mellitus y cáncer. También se buscó información sobre ensayos clínicos en ClinicalTrials.gov. En los últimos 20 años, el número de publicaciones sobre EC en SciELO fue significativamente menor en comparación con la diabetes mellitus y el cáncer. Además, solo hay dos medicamentos disponibles, a saber, benznidazol y nifurtimox, que tienen limitaciones como el número de efectos adversos. Por tanto, se han estudiado nuevas opciones terapéuticas, principalmente en el benznidazol, habiendo mostrado resultados prometedores en cuanto a optimizar la toxicidad, absorción y biodisponibilidad. En cuanto a los ensayos clínicos, se encontraron un total de siete estudios, que investigan cinco compuestos para el tratamiento de la EC. Solo uno de los compuestos aún no tiene uso clínico, el selenio, lo que sugiere una deficiencia en la búsqueda de nuevos fármacos candidatos. Esta información refuerza, por tanto, el carácter desatendido de la EC y la importancia de investigar nuevas terapias.

Palabras clave: Enfermedades desatendidas; Enfermedad de chagas; Desarrollo de medicamentos.

\section{Introdução}

O processo de desenvolvimento de um novo fármaco é bastante lento e dispendioso. Estima-se que entre 5.000 a 10.000 compostos pesquisados, apenas um consegue a aprovação de um órgão regulador, como o FDA (Food and Drug Administration). Para que esse novo fármaco tenha obtido êxito e seja aprovado para o uso em algum tratamento terapêutico, são investidos cerca de 1,5 a 2,0 bilhões de dólares, sendo necessário aproximadamente de 10 a 15 anos de pesquisa e desenvolvimento. Estima-se que cerca de metade do tempo e dinheiro requeridos em seu desenvolvimento são gastos apenas em ensaios clínicos, enquanto que a fase pré-clínica e a parte regulatória são responsáveis pela metade restante (Harrer, Shah, Antony, \& Hu, 2019).

Após a conclusão dos ensaios pré-clínicos, um medicamento para ser aprovado deve passar a próxima etapa de ensaios clínicos, que são divididos em quatro fases: I, II, III e IV. Na fase I, o fármaco é administrado em um pequeno número de voluntários saudáveis e/ou doentes, sendo determinada a dose máxima tolerável. Em relação à fase II, o número de voluntários é um pouco maior que na anterior, e tem como objetivo verificar atividade biológica, segurança e tolerabilidade (Umscheid, Margolis, \& Grossman, 2011).

Após avaliação criteriosa, o fármaco aprovado passa para a fase III, em que se avalia eficácia e segurança. Alguns aspectos são importantes para minimizar os vieses do estudo, tais como utilização de um grupo controle, em que é administrado o placebo nas mesmas condições do grupo que recebeu o medicamento contendo o princípio ativo. Para ambos os grupos, o ideal é que os pacientes sejam aleatoriamente distribuídos e que a identidade de quem recebeu o medicamento e o placebo sejam preservadas. Desta forma, é esperado que nesta fase o estudo seja conduzido em, pelo menos um estudo duplocego, ou seja, nem o paciente nem o pesquisador sabem qual tratamento foi administrado. Caso o fármaco seja aprovado, os responsáveis pelo desenvolvimento já podem solicitar o alvará para produção e comercialização de medicamento e o composto passa para a fase IV, a qual visa monitorar, entre outras coisas, efeitos adversos menos comuns (Umscheid, Margolis, \& Grossman, 2011). 
Em meio a isso, existem as chamadas Doenças Tropicais Negligenciadas (DTNs), um grupo de 17 doenças que afetam mais de 1 bilhão de pessoas, principalmente as que residem em países subdesenvolvidos (Who, 2020a). Esse grupo de doenças recebe essa denominação em virtude do pouco interesse no desenvolvimento de novos fármacos, se comparado com outras enfermidades (Weng, Chen, \& Wang, 2018).

A Doença de Chagas (DC) ou tripanossomíase americana, uma das DTNs, é uma parasitose causada pelo Trypanosoma cruzi, um protozoário que é transmitido pela picada de insetos da subfamília Triatominae, além de possuir outras formas de transmissão. Estima-se que entre 6 e 7 milhões de pessoas em todo o mundo estejam infectadas pelo T. cruzi, enquanto cerca de 75 milhões vivem em áreas de risco de infecção. Apesar da DC ser endêmica da América Latina, um número crescente de casos vem ocorrendo em países como Estados Unidos, Canadá, bem como em muitos países europeus e alguns países da África, Mediterrâneo Oriental e Pacífico Ocidental (Who, 2020b).

No Brasil, estimativas indicam que até o ano de 2015 o número de infectados pelo T. cruzi varia de 1,9 milhão a 4,6 milhões (Dias et al., 2016). Além disso, foram registrados 2.708 casos confirmados referentes a indivíduos com a forma aguda da doença no país, entre os anos de 2007 a 2018 (Alencar et al., 2020).

Para se ter uma noção do impacto da DC no Brasil, um estudo com base em dados da carga global de doenças, analisou o impacto de doze DTNs endêmicas/autóctones no país, entre 1990 e 2016. Apesar das limitações do estudo, os autores mostraram que a DC deteve os maiores registros de anos de vida ajustados pela incapacidade (DALYs - do inglês Disability-Adjusted Life Years) (141.640 DALYs [Intervalo de Incerteza 95\%: 129.065-155.941] e taxa de DALY padronizada por idade de 70,69/100.000 habitantes [Intervalo de Incerteza 95\%: 64,49-77,81]) dentre todas as DTNs estudadas (MartinsMelo et al., 2018).

Diante disso, o objetivo deste trabalho é verificar a farmacoterapia atual, bem como analisar se há interesse na busca de novos fármacos para o tratamento da DC, ou mesmo no desenvolvimento de novas alternativas terapêuticas.

\section{Metodologia}

Trata-se de uma revisão de literatura do tipo narrativa de natureza qualitativa baseada na análise da literatura publicada, artigos de revistas impressas e ou eletrônicas, na interpretação e análise crítica pessoal dos autores (Pereira, Shitsuka, Parreira, \& Shitsuka, 2018). Nesse sentido, foram realizadas buscas no PubMed, LILACS e SciELO, considerando o período de 2015 a 2020 para se obter os dados do quantitativo de trabalhos publicados relacionados aos descritores da busca nas bases de dados. Para tanto, foram utilizados os descritores "Doença de Chagas", "terapia farmacológica" e "tecnologia farmacêutica", tanto na língua inglesa quanto na língua portuguesa, sendo empregado o operador booleano "AND”. De forma análoga a metodologia utilizada por Araújo, Silva, Cândido, Silva, \& Andrade Júnior, 2020, as teses, dissertações, editoriais, trabalhos de conclusão de curso, monografias e revisões de literatura foram excluídos. Complementarmente, foram consultadas informações no DrugBank (https://go.drugbank.com/) e no site da Agência Nacional de Vigilância Sanitária (ANVISA) (https://www.gov.br/anvisa/pt-br).

Em uma primeira etapa foram pesquisados os artigos relacionados a Doença de Chagas e nas etapas subsequentes foram utilizados os buscadores para diabetes mellitus e câncer nas mesmas condições utilizadas para a Doença de Chagas para que se possa realizar a análise dos dados e assim realizar a análise entre uma doença considerada negligenciada pela indústria farmacêutica e doenças onde a indústria farmacêutica demonstra interesse no desenvolvimento de alternativas terapêuticas.

Considerando que o número de artigos publicados é um indicador do interesse sobre um determinado assunto, foi feita uma análise do número de publicações no SciELO, em um período de sete décadas (1950-2019), a fim de ter um parâmetro de 
comparação em relação às DTNs. Foram analisadas as publicações sobre a DC, diabetes mellitus e câncer. Para a realização das buscas, foi utilizado o nome de cada doença em inglês.

Adicionalmente, foi feita uma consulta no site ClinicalTrials.gov (https://www.clinicaltrials.gov/), uma base de dados norte-americana sobre estudos clínicos. A busca foi realizada no dia 20 de novembro de 2020, sendo utilizado o termo "Chagas disease" no campo "Condition or disease". Foram incluídos ensaios nas fases clínicas I, II e III, em que a intervenção fosse um agente químico e que tivessem um dos seguintes status: recrutando; ativo, mas não recrutando; inscrição por convite; ainda não está recrutando. Não foram incluídos ensaios concluídos, encerrados, suspensos, desconhecidos ou retirados devido à falta de informações (Cummings, Lee, Riitter, \& Zhong, 2018).

\section{Resultados e Discussão}

A Tabela a seguir mostra o levantamento no buscador de artigos científicos SciELO sobre o número de trabalhos publicados em relação à Doença de Chagas, diabetes mellitus e câncer em geral, em um período de sete décadas.

Tabela 1. Número de estudos publicados no SciELO utilizando os buscadores "Doença de Chagas", "diabetes mellitus" e “câncer", nos últimos 70 anos.

\begin{tabular}{lcccccccc}
\hline Doenças & $\mathbf{1 9 5 0 -}$ & $\mathbf{1 9 6 0 -}$ & $\mathbf{1 9 7 0 -}$ & $\mathbf{1 9 8 0 -}$ & $\mathbf{1 9 9 0 -}$ & $\mathbf{2 0 0 0 -}$ & $\mathbf{2 0 1 0 -}$ & \multirow{2}{*}{ Total } \\
& $\mathbf{1 9 5 9}$ & $\mathbf{1 9 6 9}$ & $\mathbf{1 9 7 9}$ & $\mathbf{1 9 8 9}$ & $\mathbf{1 9 9 9}$ & $\mathbf{2 0 0 9}$ & $\mathbf{2 0 1 9}$ & \\
\hline Doença de Chagas & 18 & 27 & 84 & 237 & 486 & 1.036 & 1.393 & 3.281 \\
Diabetes Mellitus & 1 & 2 & 3 & 15 & 203 & 2.188 & 5.095 & 7.507 \\
Câncer & 6 & 2 & 5 & 40 & 485 & 7.121 & 12.635 & 20.294 \\
\hline
\end{tabular}

Fonte: Scielo (2020).

Verifica-se que o número de estudos sobre a DC manteve um comportamento crescente ao longo das décadas. O volume de publicação sobre a DC foi substancialmente superior no período de 1950 a 1989 em comparação com diabetes mellitus e câncer. Nas últimas duas décadas, entretanto, observou-se uma mudança drástica no volume de publicação, de forma que na última década, por exemplo, a quantidade de publicações sobre câncer foi cerca de nove vezes superior e a de diabetes quase quatro vezes superior em relação à DC. Isso sugere que a DC não vem despertando muito o interesse dos pesquisadores, o que impacta no surgimento de novas formas de tratamento para a mesma.

\subsection{Tratamento farmacológico atual}

Atualmente existem dois medicamentos no mercado utilizados na farmacoterapia da DC, o benznidazol e nifurtimox. O benznidazol é o medicamento de primeira escolha para ser utilizado na terapia da doença durante a fase aguda, sendo mais tolerado por longos períodos de tempo, possui uma meia vida de eliminação entre dez e treze horas, sua biodisponibilidade oral chega a ser maior que $90 \%$, atua diminuindo a capacidade de replicação do parasita por meio da interação química covalente com moléculas, dentre elas o DNA do Trypanosoma cruzi (Dawidowski et al., 2020).

O nifurtimox é outro fármaco utilizado para tratar a doença, sujeito a metabolismo de primeira passagem suas concentrações séricas podem ser diminuídas drasticamente devido a esse evento, possui capacidade de atravessar a barreira hematoencefálica e placentária, sua ligação a proteínas plasmáticas é baixa $(<50 \%)$, seu metabolismo ocorre principalmente pelas enzimas nitroredutases e sua eliminação se dá principalmente pela via renal. Atua por meio da produção de espécies 
reativas de oxigênio (radicais superóxido) que são tóxicos para o parasito, entretanto, não são para o ser humano pois as quantidades produzidas pelo fármaco são neutralizadas nas células do homem devido a presença de enzimas como as peroxidases, catalases, superóxidos desmutases, dentre outras enzimas que exercem atividade antioxidante. Algumas vitaminas, como a E por exemplo, podem auxiliar a reduzirem os danos causados por essas espécies reativas (Nifurtimox DrugBank, 2020).

O benznidazol teve seu registro para tratamento da DC deferido pelo FDA em 29 de agosto de 2017, é utilizado principalmente no tratamento de crianças na faixa etária dos 2 a 12 anos e foi o primeiro fármaco a ser utilizado nos Estados Unidos para tratamento da doença (Benznidazole - DrugBank, 2020). No Brasil, o Laboratório Farmacêutico do Estado de Pernambuco (LAFEPE) é o único que tem registro na ANVISA para produção do benznidazol, que é distribuído gratuitamente pelo Sistema Único de Saúde para usuários portadores da doença (Brasil, 2020a; Lafepe, 2016).

Desenvolvido pela gigante farmacêutica Bayer, o nifurtimox teve seu registro no FDA aprovado em 6 de agosto de 2020 após demonstrar resultados promissores em ensaios clínicos de fase III. Entretanto, sua aprovação definitiva ainda está em análise a fim de verificar como será o comportamento da substância utilizada agora por uma parcela maior de pessoas. No Brasil, este fármaco ainda não teve seu registro solicitado por nenhuma indústria farmacêutica à vigilância sanitária para produção (Nifurtimox - DrugBank, 2020).

Ambos os fármacos mencionados são os únicos disponíveis no mercado para o tratamento da DC, cenário este que denota a escassez no tratamento farmacológico da mesma. Em adição a isso, a persistência de eventos adversos é bastante pronunciada em ambos os fármacos, mas, principalmente no caso do nifurtimox, dificultando ainda mais o tratamento do indivíduo acometido (Nifurtimox - Drugbank, 2020; Benznidazole - DrugBank, 2020).

\subsection{Desenvolvimento de novas alternativas terapêuticas}

As informações sobre alternativas terapêuticas em estudo estão representadas no Quadro 1.

Quadro 1. Artigos selecionados após a busca no PubMed, LILACS e SciELO, considerando o período de 2015 a 2020.

\begin{tabular}{|c|c|c|}
\hline Autor e ano & Medicamento & Forma farmacêutica \\
\hline García et al. (2018) & Benznidazol & $\begin{array}{c}\text { Sistemas de distribuição de drogas multiparticuladas } \\
\text { compostos por complexos interpolieletrólitos com } \\
\text { base em diferentes portadores de polimetacrilato } \\
\text { carregados de Benznidazol }\end{array}$ \\
\hline $\begin{array}{c}\text { García, Manzo e Jimenez- } \\
\text { Kairuz (2018) }\end{array}$ & Benznidazol & $\begin{array}{c}\text { Complexos interpolieletrolíticos multiparticulados à } \\
\text { base de quitosana e ácido algínico carregados com } \\
\text { Benznidazol }\end{array}$ \\
\hline Simonazzi et al. (2018) & Benznidazol & $\begin{array}{c}\text { Dispersões sólidas usando Poloxamer 407 como } \\
\text { transportador }\end{array}$ \\
\hline Souza et al. (2017) & Benznidazol & Soluções sem álcool de Benznidazol em pH neutro \\
\hline
\end{tabular}




\begin{tabular}{|c|c|c|}
\hline Alves-Silva et al. (2016) & Benznidazol & $\begin{array}{c}\text { Grânulos (pellets) preparados por extrusão e } \\
\text { esferonização para liberação imediata }\end{array}$ \\
\hline Branquinho et al. (2017) & Lychnopholide (LYC) & $\begin{array}{c}\text { Nanocápsulas (NC) convencionais preparadas a partir } \\
\text { de poli-e-caprolactona (PCL) e comparados com os } \\
\text { NC PEGuilados preparados a partir de poli (D, L- } \\
\text { lactida)-bloco-poli (etilenoglicol) (PLA-PEG) } \\
\text { projetado para depuração de sangue retardada }\end{array}$ \\
\hline Mello et al. (2016) & Lychnopholide (LYC) & $\begin{array}{c}\text { Avaliou o uso de LYC-NC oral na fase aguda e } \\
\text { também comparou com LYC-NC por via oral e } \\
\text { intravenosa na fase crônica em ratos }\end{array}$ \\
\hline $\begin{array}{c}\text { Olbuquerque e Marchetti } \\
\text { (2017) }\end{array}$ & Ácido Ursólico & $\begin{array}{c}\text { Nanoemulsão contendo ácido ursólico foi } \\
\text { desenvolvida, avaliada através de diagramas de fase } \\
\text { pseudo-ternários e sistema de balanço hidrofílico- } \\
\text { lipofílico (HLB) }\end{array}$ \\
\hline
\end{tabular}

Fonte: Autores (2020).

Os complexos interpolieletrólitos (IPEC) formulados como sistemas de distribuição de drogas multiparticuladas (MDDS) carregados de Benznidazol (BZ), envolvem matriz ou partículas de droga revestidas com base em um ou mais polímeros transportadores para controlar a liberação do fármaco. O MDDS oferece várias vantagens sobre formas de dosagem de unidade única, devido à sua multiplicidade e tamanho reduzido, incluindo baixo risco de toxicidade sistêmica, baixo risco de dumping de dose, trânsito gastrointestinal mais uniforme e confiável. Já o IPEC, que são complexos macromoleculares obtidos pela interação de polímeros quimicamente complementares, tem a capacidade de obter uma liberação de fármaco mais sustentada do que polímeros únicos (García et al., 2018).

No estudo de García et al. (2018), o MDDS carregado com BZ baseado em novos IPECs compostos de Eudragitß biocompatível, usando métodos de fabricação simples, produziram produtos físicos e estáveis. O MDDS apresentou propriedades bioadesivas e também uma liberação estendida de BZ sensível ao pH, em que as propriedades de entrega mudam dependendo da composição dos sistemas. Foi observada a liberação multicinética de BZ para cápsulas contendo uma combinação de dois MDDS com diferentes perfis de liberação de BZ, que mostraram propriedades promissoras para melhorar a farmacoterapia da Doença de Chagas no ensaio preliminar in vivo realizado.

Os estudos in vivo, realizados por García e colaboradores (2018), mostraram que o MDDS carregado com BZ exibiu eficácia para reduzir a parasitemia, enquanto diminuía os níveis de marcadores de lesão hepática em comparação ao tratamento convencional com BZ. Assim, os sistemas de liberação de BZ mostraram propriedades promissoras e podem ser alternativas farmacêuticas interessantes para melhorar a farmacoterapia da Doença de Chagas.

Outro estudo, conduzido por García, Manzo e Jimenez-Kairuz (2018), sobre IPECs multiparticulados baseados em quitosana e ácido algínico carregados com BZ, revelou que o BZ livre foi uniformemente distribuído e apresentaram características de fluidez adequadas para a formulação de cápsula de gelatina dura. Os IPECs multiparticulados exibiram 
propriedades de mucoadesão e os perfis de liberação revelaram modulação da liberação de BZ, dependendo do meio de liberação, de acordo com a absorção de fluido. Assim, os IPECs carregados com BZ atuam como um reservatório para o princípio ativo e podem ser usados para projetar sistemas de liberação bioadesivos e controlados.

O benznidazol apresenta baixa solubilidade, limitando as possibilidades de sua formulação. Uma das alternativas para melhorar o seu biodesempenho é o uso de dispersões sólidas (SDs), que produzem uma solução saturada ou supersaturada, levando à dissolução imediata de uma porção do fármaco, que fica disponível para rápida absorção, ao entrar em contato com o fluido gastrointestinal. Por outro lado, o excesso de fármaco pode precipitar no fluido gastrointestinal em partículas muito finamente divididas. Estas características frequentemente resultam em absorção de fármaco notavelmente melhorada de um SD em comparação com um comprimido convencional ou formulação de cápsula (Simonazzi et al., 2018).

Simonazzi et al. (2018), realizaram um estudo sobre essas dispersões sólidas usando Poloxamer 407 como transportador, o SD manteve a eficácia do BZ em relação à sua atividade antitripanocida. Consideraram ainda, que esse sistema pode ser uma plataforma eficaz para liberação imediata por administração oral, pois permite uma maior solubilidade cinética em água e uma taxa de dissolução mais rápida. Isso poderia resultar em uma melhor absorção e aumento da biodisponibilidade, melhorando assim o comportamento biofarmacêutico do BZ.

Souza et al. (2017), desenvolveram soluções de BZ sem álcool usando PEG 400, propilenoglicol (PPG) e água como solventes selecionados. Os resultados obtidos no estudo revelam uma relação direta entre a concentração de PEG 400 e a solubilidade de BZ. O PPG provou ser adequado como veículo líquido quando usado até $1000 \mathrm{mg} / \mathrm{kg}$ e, embora existam algumas preocupações sobre o uso de PPG em altas concentrações, há uma variedade de formulações comerciais licenciadas contendo esse glicol.

Além disso, os autores do estudo observaram que a solubilidade aquosa de $\mathrm{BZ}$ foi aumentada de $0,23 \mathrm{mg} / \mathrm{ml}$ para $18,38 \mathrm{mg} / \mathrm{ml}$ (80 vezes). A formulação permaneceu estável a $4 \pm 2{ }^{\circ} \mathrm{C}, 25 \pm 2{ }^{\circ} \mathrm{C}$ e $45 \pm 2{ }^{\circ} \mathrm{C}$, com teor de fármaco adequado e sem variabilidade significativa. Os excipientes incluídos na formulação final cobriram efetivamente o sabor amargo do fármaco. Já os estudos in vivo confirmaram a adequação da formulação desenvolvida de BZ para reduzir a parasitemia e a mortalidade, particularmente na dose de $40 \mathrm{mg} / \mathrm{kg} / \mathrm{dia}$ (Souza et al., 2017).

Normalmente, o BZ é comercializado utilizando a forma farmacêutica comprimido, onde estes são desenvolvidos sem nenhum aprimoramento tecnológico, como o controle de liberação de BZ. Tendo em vista a necessidade de fabricar alternativas terapêuticas versáteis, elaboradas contendo os princípios ativos atualmente utilizados para tratamento da DC, como o próprio BZ e derivados azólicos, foi realizado estudo por Alves-Silva e colaboradores (2016) no qual foi desenvolvida uma nova forma farmacêutica (FF), denominada "Pellets" (grânulos), através da técnica de extrusão-esferonização.

Após experimentos de mistura de insumos farmacêuticos, Alves-Silva e colaboradores (2016) observaram a redução do tempo de desintegração e aumento da dissolução do fármaco de acordo com as composições qualitativas e quantitativas dos agentes desintegrantes, logo, a baixa solubilidade em água foi trabalhada, viabilizando possível alternativa farmacológica em pacientes pediátricos cuja a titulação da dosagem é necessária e geralmente é realizada dividindo-se os comprimidos em pedaços, o que resulta em baixa precisão, podendo interferir no tratamento da doença.

Um novo candidato a fármaco tem sido estudado, metabólito denominado Lychnopholide (LYC), sendo uma lactona sesquiterpênica lipofílica isolada de Lychnophora trichocarpha (Spreng.), apresentando uma ampla gama de efeitos farmacológicos, incluindo atividade contra tripanosomatídeos. Com relação a forma farmacêutica empregada, a habilidade de nanocápsulas (NC) poliméricas em aprimorar as propriedades biofarmacêuticas de substâncias lipofílicas, tem sido amplamente demonstrada (Branquinho et al., 2017; Mello et al., 2016). 
Estudo realizado por Branquinho e colaboradores (2017) relata que LYC na formulação de NC foi capaz de curar camundongos infectados experimentalmente com cepas de T. cruzi sensíveis, parcialmente resistente e resistente ao benznidazol, nas fases aguda e crônica da doença. Tal eficácia nunca havia sido alcançada por qualquer outro medicamento/formulação até o momento.

Esse achado, pôde ser comparado com a ação de NC, uma vez que possuindo na superfície cadeias hidrofílicas ligadas covalentemente de polietilenoglicol (PEG), existem mais chances de entregar substâncias lipofílicas, como lychnopholide para os parasitas tripomastigotas do sangue que prevalecem na fase aguda da infecção. Além disso, NC PEGuilada pode se acumular nas células musculares do coração e do esôfago, onde amastigota na forma intracelular do parasita desencadeia cardiomiopatia grave e danos na fase crônica da DC (Branquinho et al., 2017).

Estudo recente realizado por Mello e colaboradores (2016), também evidenciou os benefícios da NC (mesma formulação), comparando e avaliando o uso nas diferentes fases da infestação, como também tratados por administração intravenosa durante a fase aguda, apesar da via oral ser preferida para tratamento de infecções crônicas. Quando o LYC é administrado por via intravenosa como uma solução (Free-LYC), a parasitemia foi reduzida e a taxa de sobrevivência aumentou quando comparada ao grupo não tratado na fase aguda da infecção em camundongos, embora nenhuma cura tenha sido observada.

Neste contexto, pesquisas vem dando destaque a outro agente promissor com ação tripanocida, denominado ácido ursólico. Este por sua vez, é um terpeno natural hidrofóbico, fator que limita a biodisponibilidade impactando sua eficácia, o qual foi incorporado em sistema nanoestruturado, sendo mais adequado na distribuição de fármacos lipofílicos. Sendo testada nanoemulsões, que correspondem a dispersões finas de óleo em água ou água em óleo sendo sistemas cineticamente estáveis, possuindo ampla aplicação nas indústrias cosmética e farmacêutica devido às suas propriedades vantajosas (Oliveira, Carneiro, Albuquerque, \& Marchetti, 2017).

Dentre os experimentos e testes realizados, vale a pena destacar os de citotoxicidade e tripanocida, que demonstraram que o sistema desenvolvido tinha atividade contra as formas replicantes do parasita (amastigotas) e que era seguro por apresentar elevado CC50 (citotoxicidade). É importante ressaltar que esse valor foi superior ao do benznidazol, mostrando que o ácido ursólico nanoemulsificado foi mais seguro do que o medicamento padrão no tratamento da Doença de Chagas, logo as amostras testadas podem ser boas candidatas para experimentos in vivo e têm maior probabilidade de apresentar bons resultados (Oliveira, Carneiro, Albuquerque, \& Marchetti, 2017).

\subsection{Compostos em fase clínica}

O Quadro 2 mostra os ensaios clínicos registrados no site ClinicalTrials.gov, no dia 20 de novembro de 2020. 
Research, Society and Development, v. 10, n. 2, e21110212419, 2021

(CC BY 4.0) | ISSN 2525-3409 | DOI: http://dx.doi.org/10.33448/rsd-v10i2.12419

Quadro 2. Ensaios clínicos (n=7) selecionados no site ClinicalTrials.gov.

\begin{tabular}{|c|c|c|c|c|c|c|}
\hline Intervenção & Fase & Mascaramento & $\begin{array}{c}\text { Estimativa de } \\
\text { participantes } \\
\text { recrutados } \\
\end{array}$ & Status & Financiamento & ID Clinicaltrials.gov \\
\hline Benznidazol & 3 & Aberto (Open Label) & 164 & Recrutando participantes & Insud Pharma & NCT04024163 \\
\hline Nifurtimox & 3 & Quadruplo-cego & 330 & $\begin{array}{c}\text { Ativo, mas não recrutando } \\
\text { participantes }\end{array}$ & Bayer & NCT02625974 \\
\hline Selênio & 3 & Duplo-cego & 130 & Recrutando & $\begin{array}{c}\text { Fundação Oswaldo } \\
\text { Cruz }\end{array}$ & NCT00875173 \\
\hline $\begin{array}{l}\text { Benznidazol e } \\
\text { Nifurtimox }\end{array}$ & 2 & Aberto & 450 & Recrutando & $\begin{array}{l}\text { University of Texas, } \\
\text { El Paso }\end{array}$ & NCT03981523 \\
\hline Colchicina & 2 & Triplo-cego & 60 & Recrutando & $\begin{array}{c}\text { Hospital Geral da } \\
\text { Universidade de São } \\
\text { Paulo }\end{array}$ & NCT03704181 \\
\hline Benznidazol & 3 & Quadruplo-cego & 600 & Recrutando & $\begin{array}{c}\text { Tulane University } \\
\text { School of Public } \\
\text { Health and Tropical } \\
\text { Medicine }\end{array}$ & NCT03672487 \\
\hline Amiodarona & 3 & Quadruplo-cego & 200 & Recrutando & $\begin{array}{l}\text { Fundación } \\
\text { Cardioinfantil } \\
\text { Instituto de } \\
\text { Cardiología }\end{array}$ & NCT03193749 \\
\hline
\end{tabular}

Fonte: ClinicalTrials (2020). 
Ao todo, 5 compostos estão em fase clínica para tratamento da DC. Um estudo sobre tratamentos para Alzheimer analisou ensaios clínicos registrados no mesmo site, no dia 30 de janeiro de 2018. Os autores obtiveram um total 112 compostos distribuídos nas fases I, II e III (Cummings, Lee, Riitter, \& Zhong, 2018), resultado esse bem superior ao número registrado para a DC, o que sugere um interesse mais amplo na busca por tratamentos para o Alzheimer.

Dos compostos estudados, apenas o selênio não é um fármaco já registrado para tratamento de outra condição. Além disso, em quatro dos sete ensaios clínicos, os compostos estudados foram benznidazol e nifurtimox, os quais foram lançados no começo da década de 1970 e permanecem como os únicos utilizados na clínica (Bermudez, Davies, Simonazzi, Real, \& Palma, 2015).

A colchicina e a amiodarona, dois dos compostos estudados, já possuem indicação terapêutica descrita, sendo elas, respectivamente, crises agudas de gota e arritmias cardíacas (Brasil, 2020b). É importante salientar que, no caso da colchicina, o ensaio clínico teve o objetivo de avaliar o potencial de tratar a inflamação do miocárdio em pacientes com a DC, e não o combate ao parasita. Apesar disso, o emprego desses dois compostos evidencia a importância do reposicionamento de fármacos, uma estratégia que, diferentemente do processo tradicional, proporciona maior economia de tempo e dinheiro (Xue, Li, Xie, \& Wang, 2018).

Quanto à origem dos recursos para a realização dos estudos, nota-se que na maior parte dos mesmos o financiamento foi proveniente de instituições sem fins lucrativos. Tal cenário evidencia a falta de interesse por parte da indústria farmacêutica em desenvolver novos fármacos para a DC, além de que, no caso de universidades e outras entidades filantrópicas, a falta de incentivo pode ser um grande desafio para a realização de pesquisas (Cruz et al., 2016).

\section{Considerações Finais}

A Doença de Chagas permanece sendo um desafio no tocante à busca de novas estratégias terapêuticas. Apesar do crescimento no número de estudos ao longo de 70 anos, esta parasitose tem sido menos visada por pesquisadores nos últimos 20 anos, em comparação com diabetes mellitus e câncer.

Vale destacar que há apenas duas opções de medicamentos disponíveis atualmente, sendo elas benznidazol e nifurtimox. Estes possuem eficácia limitada a depender do estágio da doença, além de possuírem muitos efeitos adversos. Estudos recentes têm buscado novas alternativas que amenizem tais problemas, principalmente no caso do benznidazol. Tais estudos mostraram melhorias relevantes em determinados aspectos, como controle da liberação, redução de toxicidade sistêmica, otimização da absorção e maior biodisponibilidade, mascaramento do sabor do fármaco, redução do tempo de desintegração, entre outros.

Com relação ao desenvolvimento de novos fármacos, há poucos ensaios clínicos nas fases I, II e III, além de que na maior parte dos mesmos os fármacos estudados são o benznidazol e o nifurtimox, já utilizados no tratamento da doença há décadas. Vale pontuar que alguns fármacos já aprovados para tratar outras doenças têm sido estudados, o que pode tornar mais barato o custo da pesquisa. Dos candidatos, apenas o selênio ainda não possui nenhuma indicação clínica registrada.

Tal cenário mostra a necessidade de mais investimentos por parte da indústria farmacêutica, bem como mais incentivos a instituições sem fins lucrativos, a fim de buscar novos medicamentos para o tratamento da DC, a qual permanece com opções terapêuticas muito limitadas, mesmo após mais de cem anos desde a sua descoberta.

É fato que existem algumas doenças negligenciadas pela indústria farmacêutica pelo fato de que o tratamento medicamentoso para estas doenças provavelmente não apresenta um bom prognóstico de retorno financeiro para a indústria farmacêutica, resultando no sofrimento da população, principalmente a que possui menos recursos financeiros. Desta forma, o estudo e desenvolvimento de novas alternativas terapêuticas, sejam elas novos medicamentos para a DC e o reposicionamento de fármacos, além da utilização de ferramentas in silico, as quais contribuem para otimizar o tempo e dinheiro investidos, são 
de grande importância para reverter a situação atual.

\section{Referências}

Alencar, M. M. F., Santos Filho, R. A. B., Hirschheiter, C. Â., Carmo, M. C. N., Santana, M. S., Ramos, J. L. D., \& Galvão, P. V. M. (2020). Epidemiologia da Doença de Chagas aguda no Brasil de 2007 a 2018. Research, Society and Development, 9(10), e8449109120-e8449109120. https://doi.org/10.33448/rsdv9i10.9120.

Agência Nacional de Vigilância Sanitária. (2020b). Bulário eletrônico. <https://consultas.anvisa.gov.br/\#/bulario/>.

Agência Nacional de Vigilância Sanitária. (2020a). Câmara de Regulação do Mercado de Medicamentos-

CMED. <http://antigo.anvisa.gov.br/documents/374947/6048620/LISTA_CONFORMIDADE_2020_10_v1.pdf/7b88a38f-1b2f-4768-b589-f62b4beb1762>.

Alves-Silva, I., Marreto, R. N., Gelfuso, G. M., Sá-Barreto, L. C. L., Lima, E. M., \& Cunha-Filho, M. S. S. (2016). Preparation of benznidazole pellets for immediate drug delivery using the extrusion spheronization technique. Drug Development and Industrial Pharmacy, 43(5), 762-769. http://dx.doi.org/10.1080/03639045.2016.1220574.

Araújo, J. M. D., Silva, A. P., Cândido, M. B., Silva, T. W. M., \& Andrade Júnior, F. P. (2020). Estudo etnofarmacológico de Anacardium occidentale: uma breve revisão. Research, Society and Development, 9(8), e487985802-e487985802. http://dx.doi.org/10.33448/rsd-v9i8.8502.

Bermudez, J., Davies, C., Simonazzi, A., Real, J. P., \& Palma, S. (2016). Current drug therapy and pharmaceutical challenges for Chagas disease. Acta Tropica, 156, 1-16. http://dx.doi.org/10.1016/j.actatropica.2015.12.017.

Branquinho, R. T., Pound-Lana, G., Milagre, M. M., Saúde-Guimarães, D. A., Vilela, J. M. C., Andrade, M. S., \& Mosqueira, V. C. F. (2017). Increased Body Exposure to New Anti-Trypanosomal Through Nanoencapsulation. Scientific Reports, 7(1). https://doi.org/10.1038/s41598-017-08469-X.

Cruz, C. A. B., Silva, A. L. S., Alencar, E. M. D., Santos, N. J. B., Moreira, J. J. S., Paixão, A. E. A., \& Gomes, I. M. A. (2016). Tecnologias que empregam fármacos antiparasitários para tratamento da doença Chagas. Revista Eletrônica de Comunicação, Informação e Inovação em Saúde, 10(1), 1-9. https://doi.org/10.29397/reciis.v10i1.1075

Cummings, J., Lee, G., Riitter, A., \& Zhong, K. (2018). Alzheimer's disease drug development pipeline: 2018. Alzheimer's \& Dementia: Translational Research \& Clinical Interventions, 4, 195-214. https://doi.org/10.1016/j.trci.2018.03.009.

Dawidowski, M., Kalel, V. C., Napolitano, V., Fino, R., Schorpp, K., Emmanouilidis, L., ... \& Popowicz, G. M. (2020). Structure-Activity Relationship in Pyrazolo[4,3 c]pyridines, First Inhibitors of PEX14-PEX5 Protein-Protein Interaction with Trypanocidal Activity. Journal of Medicinal Chemistry, 63, 847-879. https://doi.org/10.1021/acs.jmedchem.9b01876.

Dias, J. C. P., Ramos Jr, A. N., Gontijo, E. D., Luquetti, A., Shikanai-Yasuda, M. A., Coura, J. R., ... \& Alves, R. V. (2016). Consenso Brasileiro em Doença de Chagas, 2015. Epidemiologia e Serviços de Saúde, 25(esp.), 7-86. https://doi.org/10.5123/S1679-49742016000500002.

DrugBank. 2020. Benznidazole - DrugBank. <https://go.drugbank.com/drugs/DB11989>.

DrugBank. 2020. Nifurtimox - DrugBank. <https://go.drugbank.com/drugs/DB11820>.

García, M. C., Manzo, R. H., \& Jimenez-Kairuz, A. (2018). Polysaccharides-based multiparticulated interpolyelectrolyte complexes for controlled benznidazole release. International Journal of Pharmaceutics, 545(1-2), 366-377. https://doi.org/10.1016/j.ijpharm.2018.05.017.

García, M. C., Martinelli, M., Ponce, N. E., Sanmarco, L. M., Aoki, M. P., Manzo, R. H., \& Jimenez-Kairuz, A. F. (2018). Multi-kinetic release of benznidazole-loaded multiparticulate drug delivery systems based on polymethacrylate interpolyelectrolyte complexes. European Journal of Pharmaceutical Sciences, 120, 107-122. https://doi.org/10.1016/j.ejps.2018.04.034.

Harrer, S., Shah, P., Antony, B., \& Hu, J. (2019). Artificial intelligence for clinical trial design. Trends in Pharmacological Sciences, 40(8), 577-591. https://doi.org/10.1016/j.tips.2019.05.005.

Lafepe, Laboratório Farmacêutico do Estado de Pernambuco. (2016). Guia de Compras do Benznidazol. <http://www.lafepe.pe.gov.br/wpcontent/uploads/2016/08/Guia-de-Compras-BZD-27082010_revis\%C3\%A3o-Ago16.pdf>.

Martins-Melo, F. R., Carneiro, M., Ramos Jr, A. N., Heukelbach, J., Ribeiro, A. L. P., \& Werneck, G. L. (2018). The burden of neglected tropical diseases in Brazil, 1990-2016: a subnational analysis from the Global Burden of Disease Study 2016. PLoS Neglected Tropical Diseases, 12(6), e0006559. https://doi.org/10.1371/journal.pntd.0006559.

Mello, C. G. C., Branquinho, R. T., Oliveira, M. T., Milagre, M. M., Mosqueira, V. C. F., \& Lana, M. (2016). Efficacy of lychnopholide polymeric nanocapsules after oral and intravenous administration in murine experimental Chagas disease. Antimicrobial Agents and Chemotherapy, 60(9), 5215-5222. https://doi.org/10.1128/AAC.00178-16.

Oliveira, E. C. V., Carneiro, Z. A., Albuquerque, S., \& Marchetti, J. M. (2017). Development and Evaluation of a Nanoemulsion Containing Ursolic Acid: a Promising Trypanocidal Agent. AAPS PharmSciTech, 18(7), 2551-2560. https://doi.org/10.1208/s12249-017-0736-y.

Pereira A. S., Shitsuka, D. M., Parreira, F. J., \& Shitsuka, R. (2018). Metodologia da pesquisa científica. [e-book]. Santa Maria. Ed. UAB/NTE/UFSM. <https://repositorio.ufsm.br/bitstream/handle/1/15824/Lic_Computacao_Metodologia-Pesquisa-Cientifica.pdf?sequence=1>.

Simonazzi, A., Davies, S., Cid, A. G., Gonzo, E., Parada, L., \& Bermúdez, J. M. (2018). Preparation and Characterization of Poloxamer 407 Solid Dispersions as an Alternative Strategy to Improve Benznidazole Bioperformance. Journal of Pharmaceutical Sciences, $107,2829-2836$. https://doi.org/10.1016/j.xphs.2018.06.027. 
Research, Society and Development, v. 10, n. 2, e21110212419, 2021

(CC BY 4.0) | ISSN 2525-3409 | DOI: http://dx.doi.org/10.33448/rsd-v10i2.12419

Souza, H. F. S., Real, D., Leonardi, D., Rocha, S. C., Alonso, V., Serra, E., ... \& Salomon, C. J. (2017) Development and in-vitro/in-vivo evaluation of a novel benznidazole liquid dosage form using a quality-by-design approach. Tropical Medicine \& International Health, 22(12), 1514-1522. https://doi.org/10.1111/tmi.12980.

Umscheid, C. A., Margolis, D. J., \& Grossman, C. E. (2011). Key concepts of clinical trials: a narrative review. Postgraduate Medicine, 123(5), 194-204. https://doi.org/10.3810/pgm.2011.09.2475.

Xue, H., Li, J., Xie, H., \& Wang, Y. (2018). Review of drug repositioning approaches and resources. International Journal of Biological Sciences, 14(10), 1232-1244. https://doi.org/10.7150/ijbs.24612.

Weng, H.-B., Chen, H.-X., \& Wang, M.-W. (2018). Innovation in neglected tropical disease drug discovery and development. Infectious Diseases of Poverty, 7(67), 1-9. https://doi.org/10.1186/s40249-018-0444-1.

World Health Organization. (2020a). Neglected Tropical Diseases. https://www.who.int/news-room/q-a-detail/neglected-tropical-diseases.

World Health Organization. (2020b). Chagas Disease. https://www.who.int/health-topics/chagas-disease\#tab=tab_1. 